It acknowledges that in certain ways professional people may advertise their existence or services to the public-for instance, a doctor's plate showing his qualifications. But it goes on from a commercial analogy to say that greater freedom to advertise in the professions may be expected to have various advantages. "New entrants might be able to build up their practices more quickly than at present," though "freedom to advertise might serve to entrench established practitioners." "The introduction of new methods and of new kinds of service might be expected to take place more quickly and at lower cost." In other words Dr. X arrives in town, advertises in the local paper that he offers the latest treatment for leukaemia, to be countered next week by an advertisement from the established firm offering an experience based on 20,000 man-hours in the treatment of bronchitis. "We doubt whether," the Commission adds from its considerable experience of the commercial world, "the cost of advertising would outweigh the greater efficiency likely to flow from more effective competition."

On the restricted access of patients to consultants through general practitioners the Commission contents itself with quotations from the B.M.A.'s Handbook ${ }^{3}$ and written evidence, ${ }^{4}$ adding the uncomprehending remark that "it has apparently been found desirable for reasons of administration to build the access rule formally into the State medical service." The great benefits this practice brings to the patient go without comment.

Having spent more than three years on the inquiry the Commission must be disappointing its sponsors when it

\section{Purulent Neonatal Meningitis}

Neonatal meningitis remains a challenging problem. There is no other month in life in which the disease is so common, and yet early diagnosis remains difficult. We usually cannot prevent it, and the results of treatment are still disappointing in spite of the increasing number of broad-spectrum antibiotics.

J. C. Overall ${ }^{1}$ in the United States recently reported the results of a prospective co-operative study in which. 14 centres took part. Over the period 1959 to 1966 they studied 54,533 live births. Twenty-five cases of neonatal meningitis occurred, an incidence of 0.46 per 1,000 , cases with congenital malformations of the central nervous system being excluded. Their figure agrees with the 0.4 per 1,000 found earlier by R. V. Groover and co-workers. ${ }^{2}$ The incidence was greater in premature infants: 1.36 per 1,000 as compared with 0.37 per 1,000 in full-term babies. This difference has long been recognized, and it is known that premature infants have a low concentration of transplacentally acquired immune anti-

\footnotetext{
1 Overall, J. C., jun., Fournal of Pediatrics, 1970, 76, 499

2 Groover, R. V., Sutherland, J. M., and Landing, B. H., New England Fournal of Medicine, 1961, 264, 1115.

3 Vahlquist, B., American Fournal of Diseases of Children, 1960, 99, 729.

- Lorber, J., Kalhan, S. C., and Mahgrefte, B., Archives of Disease in Childhood, 1970, 45, 178 .

- Yu, J.S., and Grauaug, A., Archives of Disease in Childhood, 1963, 38, 391.

- Yu, J. S., and Grauaug, A., Archivis of Disease in Childhood, 1963,

7 Watson, D. J., G., Fournal of Pediatrici, 1957, 50, 353.

Ziai, M., and Haggerty, R. J., Neu England Fournal of Medicine, 1958, 259,314 .

- Lorber, J., and Bruce, A. M., Developn 'ental Medicine and Child Neurology,

1963, 5, 146.
${ }^{10}$ Berman, P. H., and Banker, B. Q., Pedictrics, 1966, 38, 6.
}

concludes that "without more investigation than is possible in a general inquiry under section 5 of the Monopolies and Mergers Act 1965 we cannot say how far the practices of particular professions are justifiable in the light of these considerations." One reason for this inability, as the report goes on to point out, is that no profession has had an opportunity of justifying its practices to the Commission. ${ }^{5}$ Hence the recommendation for a further series of inquiries into, presumably, some 130 professions in all their variety.

Such restrictions as the medical profession exercises on entry into its ranks, on the relationships between doctor and patient and one doctor and another, and such as the State too imposes on medical men and women-all these are the subject of frequent comment and criticism in the medical press and at widely reported meetings. It is right that they should be openly discussed, since public confidence in the profession depends on the knowledge that doctors do not abuse the intensely personal trust reposed in them. Professional privileges are an expression of the rights of the patient, not of the doctor. For the profession to continue to examine these matters and to ensure that the public is fully acquainted with them can only be beneficial. But as this has for some time been its practice the advice from the Monopolies Commission may be thought superfluous.

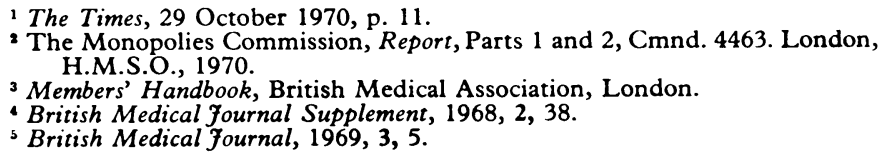

bodies, especially against Escherichia coli. ${ }^{3}$ If the unstated number of cases associated with spina bifida cystica had been added, the numbers would have been substantially increased. ${ }^{4}$

Practically any pyogenic organism may cause meningitis in the newborn, but in all larger series Gram-negative enteric organisms have predominated. $E$. coli is by far the commonest and is responsible for about one-third of all cases. ${ }^{256}$ This and other enteric organisms are especially important in the first two weeks of life, and in the series described by J. S. Yu and A. Grauaug ${ }^{5}$ they were responsible for the meningitis in 23 out of 26 patients under 2 weeks of age.

Neonatal meningitis is often part of a septicaemic process, and infection is frequently present in other parts of the body. The same organism was discovered in blood culture in 11 of 18 cases in Overall's series. Prenatal or immediately postnatal pneumonia often preceded the meningitis. Others of these babies had otitis media, gastroenteritis, or intestinal obstruction leading to peritonitis.

Maternal infection is an important predisposing cause of the disease. Ten of 25 mothers had peripartum infection, and in six of these the same strain of organism was recovered from mother and child. The infection in the mother was usually in the genitourinary tract.

Complications during labour and delivery, especially fetal distress, were also commoner than in matched controls. The infants with obstetric complications developed meningitis in the first fortnight. The importance of complications during labour and delivery was shown by Groover. ${ }^{2}$ Of 17 babies whose meningitis began before the sixth day of life 15 were born after obstetric complications. Histological study. of the 
placenta showed chorioamnionitis in $36 \%$ as compared with $11 \%$ in controls.

The early clinical picture is non-specific and usually consists of lethargy, raised or subnormal temperature, anorexia, vomiting, and irritability. The classical signs of neck stiffness or bulging fontanelle are rarely present. Signs and symptoms of grave neurological disturbance develop in the later stage of the disease: coma, convulsions, opisthotonus, and bulging fontanelle are common and of grave prognostic significance. "Blind" antibiotic therapy before lumbar puncture leads to delay in diagnosis and may render bacteriological identification impossible. Any infant who has symptoms suggestive of meningitis should have his cerebrospinal fluid examined. Blood should also be obtained for culture not only because the same organisms are found in two-thirds of all cases but because occasionally blood culture alone will give bacteriological evidence. If the cerebrospinal fluid becomes blood-stained during withdrawal and is unsuitable for cell count and biochemical test, it should nevertheless be sent for culture.

Treatment should begin as soon as these samples have been obtained. Any delay aggravates the prognosis. Antibiotic drug or drugs can be chosen according to the likely causative organism in the individual case. The initial choice might be guided by clinical features or by the immediately available result of the Gram stain on the organisms in the cerebrospinal fluid, or from any available culture of organisms from maternal infections, or from an earlier local infection of the infant. The drug regimen must be reviewed when sensitivity tests become available and changed if necessary. The choice of drugs is now large, but the published results are disappointing. Fifteen of Overall's 25 patients died. This $60 \%$ mortality rate is barely better than reported by others in larger series of cases. ${ }^{2}$ 5-8

In view of the relative rarity of the condition, the varied bacteriology, and the frequent introduction of promising new antibiotic drugs it has not been possible to determine by controlled trials which drug or combination of them is the best for an individual case.

The treatment follows no uniform plan in any of the larger series of cases reported, all of which were retrospective analyses of patients admitted over periods of many years. Chloramphenicol, streptomycin, kanamycin, and many others have been given, singly or in combination. There is no evidence that a combination of drugs is more efficient than a single one, as long as adequate bactericidal concentration of the correct drug is attained in the cerebrospinal fluid. ${ }^{4}$ There is little published information about the value of intrathecal therapy, yet most drugs appropriate for the treatment of meningitis can be given intrathecally. If intrathecal therapy is started at the time of the initial diagnostic lumbar puncture, a high antibiotic concentration in the cerebrospinal fluid can be obtained without delay, with a promise of better results.

Though it would be eminently desirable to prevent neonatal meningitis, there is little proof that this can be achieved. There are no prospective controlled trials in anatomically normal babies, and in an earlier trial in babies born with myelomeningocele the results were disappointing. ${ }^{9}$ It is known that neonatal meningitis can occur in spite of systemic antibiotic therapy. Nevertheless, it is common practice to provide antibiotic protection for those infants at the highest risk. These are the infants who have suffered fetal distress, who were born after premature rupture of the membranes or substantial obstetric complications, or whose mothers had perinatal infections, ${ }^{10}$ especially if the infant is premature.

\section{Hygiene in Food Shops}

The typhoid epidemic at Aberdeen in $1964^{1}$ almost certainly originated from a single can of corned beef, but not all the numerous victims ate meat from it. There is every reason for believing that in this and similar outbreaks other meat was contaminated from surfaces with which the infected material had been in contact, notably slicing machines but including any surface on which such food is laid. Since this disaster there has naturally been an access of interest in methods whereby contamination of one food by another can be prevented. The official report of the Aberdeen outbreak ${ }^{2}$ recommended that there should be some system of testing solutions used for cleansing and disinfection in food premises, since the use of such a solution is generally the only feasible way of removing contamination.

A study of methods for such tests, and of the merits of different reagents for this purpose, has been made by $R$. J. Gilbert. $^{3}$ The work was done in a large self-service store, and the surfaces sampled were those of a slicing machine, a carving knife, a plastic display dish, a Formica working surface, and a wooden chopping table. Two methods were used for making bacterial counts of measured areas on these surfaces. In the first an alginate swab is rubbed over the area. It is then dissolved in quarter-strength Ringer's solution containing any necessary neutralizers if the surface has been treated with disinfectant, and plate cultures are made from an aliquot of this fluid. The second is the "agar sausage" method devised by $\mathrm{L}$. Ten Cate, ${ }^{4}$ employing nutrient agar in a sausage casing $3.4 \mathrm{~cm}$. in diameter and $17 \mathrm{~cm}$. long. The end of this sausage is cut off and the flat surface applied to the area to be sampled. A slice $0.5 \mathrm{~cm}$. thick is then taken off the end and placed in a Petri dish, leaving a fresh surface for the next sampling. The sausage itself is protected from contamination by the hand holding it by its casing. By this means many areas can be simply and economically sampled in a short time. The method gives much lower counts than swabbing, but the author commends it as a routine control method for use by public health inspectors, and particularly for providing food handlers with a visual demonstration of the presence of bacteria on surfaces for which they are responsible.

The results of comparing the efficacy of different reagents were clear-cut and should be of great practical interest to public health authorities and the retail food industry. An oil intended for the cleaning of slicing machines gave generally unsatisfactory counts. The reductions in bacterial counts produced by all other treatments were substantial but very different in degree. An anionic detergent used alone was more effective than a hypochlorite solution (200 parts per million) used alone, evidently because the cleansing effect is even more important than bactericidal action. Intermediate results were given by mixtures of an anionic detergent and chlorinated trisodium phosphate or a quaternary ammonium compound. Much the largest and most consistent reductions in bacterial counts were given by applying first the anionic detergent and then the hypochlorite solution. This procedure takes a little more time, but the results fully justify it and are indeed what might be expected. The detergent cleanses the surface of grease and other material and leaves the field clear for the full and rapid bactericidal effect of hypochlorite. It is

\footnotetext{
1 British Medical fournal, 1964, 2, 1652.

2 Scottish Home and Health Department. The Aberdeen Typhoid Outbreak, Cmnd. 2542. Edinburgh, H.M.S.O., 1964.

- Ten Cate, L., fournal of Applied Bacteriology, 1965, 28, 221.
} 